\title{
GLOBAL EXPONENTIAL STABILITY FOR NONLINEAR DELAY DIFFERENTIAL SYSTEMS
}

\author{
Leonid Berezansky, brznsky@cs.bgu.ac.il \\ Ben-Gurion University of the Negev, Beer-Sheva, Israel
}

\begin{abstract}
We give a review on recent results for global stability for nonlinear functional differential equations. Such equations include delay differential equations, integro-differential equations and equations with distributed delay and are applied as mathematical models in Population Dynamics and other sciences. We also consider methods used to study global stability: constructing of Lyapunov functional, applications of special matrices such as M-matrix or special matrix functions such as matrix measure, method of matrix inequalities, which is very popular in papers on Control Theory, fixed point approach and using a notion of nonlinear Volterra operator.

Keywords: the global stability, the Lyapunov functional, the matrix measure, the method of matrix inequalities, the nonlinear Volterra operator.
\end{abstract}

\section{INTRODUCTION}

One of the main motivations to study nonlinear delay differential systems is their importance in investigations of artificial neural network models and more generally in Mathematical Biology.

In this review paper we will discuss a global stability problem for linear and nonlinear systems of FDE. Such investigations one can divide by the form of a system: vector o scalar form and also by the method of investigation. The main methods are: constructing of Lyapunov functionals, applications of special matrices such as M-matrix or special matrix functions such as matrix measure, method of matrix inequalities, which is very popular in papers on Control Theory, fixed point approach and using a notion of nonlinear Volterra operator.

In this paper we consider all forms of systems - vector and scalar forms and some of methods - applications of M-matrix and matrix measure, and using an abstract Volterra (causal) operator.

Some worlds about other methods. Lyapunov functionals are usually used for concrete systems arising in applications or for general systems with results formulated in implicit form. In method of matrix inequalities they use very unwieldy matrices, fixed point approach is new and I don't now interesting results, obtained by this method for systems of FDE.

\section{VECTOR FORM SYSTEM}

\subsection{Matrix Measure}

Denote by $\|x\|$ a vector norm in $\mathbb{R}^{n}$. Matrix measure(logarithmic matrix norm) can be denoted by the following equality

$\mu_{A}(t)=\lim _{\epsilon \rightarrow 0^{+}} \frac{\|I+\epsilon A(t)\|-1}{\epsilon}$.

Application of the matrix measure is one of the main tools in investigations of stability for systems of ODE. For FDE the matrix measure was also applied, see for example [1].

In the recent paper [2] the authors considered the system

$\frac{d x}{d t}=A x(t)+F\left(t, x\left(t-\tau_{1}\right), \ldots, x\left(t-\tau_{m}\right), t>t_{0} \geq 0\right.$,

and obtained the following result.

Theorem. Suppose

$\left\|F\left(t, u_{1}, \ldots, u_{m}\right)\right\| \leq \sum_{k=1}^{m} \lambda_{k}\left\|u_{k}\right\|, \sum_{k=1}^{m} \lambda_{k}<-\mu_{A}$.

Then Eq. (2.1) is globally exponentially stable.

In paper [3] we extended and improved this result.

Consider the following system

$\frac{d x}{d t}=A(t) x(t)+F\left(t, x\left(h_{1}(t)\right), \ldots, x\left(h_{m}(t)\right), t>t_{0} \geq 0\right.$, 


\section{Краткие сообщения}

where $A(t) \in \mathbb{R}^{n \times n}$ is a measurable locally essentially bounded matrix function and $F\left(t, u_{1}, \ldots, u_{m}\right)$ is a Caratheodory function, i.e., it is a locally essentially bounded matrix function of $t$, and continuous matrix function at each point $\left(u_{1}, \ldots, u_{m}\right)$ for any $t, h_{k}$ are measurable functions, and $0 \leq t-h_{k}(t) \leq \tau$, $(k=1,2, \ldots, m)$; with the initial value problem

$$
x(t)=\varphi(t), t<t_{0}, x\left(t_{0}\right)=x_{0},
$$

where $\varphi$ is a continuous function.

Definition 2.1. The solution of system (2.2)-(2.3) is a locally absolutely continuous function for $t \geq t_{0}$, that satisfied equality (2.2) almost everywhere for $t \geq t_{0}$ and initial conditions (2.3) for $t \leq t_{0}$.

Assume that the unique global solution for system (2.2)-(2.3) exists. Now we want to give the definitions of the global stability.

Definition 2.2. The open set $Q \subset \mathbb{R}^{n}$ attracts all solutions of (2.2)-(2.3) with the initial conditions in the open set $Q_{0} \subset \mathbb{R}^{n}$ if for any $t_{0} \geq 0$ and any solution $x$ of problem (2.2)-(2.3) with $\varphi(t) \in Q_{0}, x_{0} \in Q_{0}$ there exists $t_{1} \geq t_{0}$ such that $x(t) \in Q, t \geq t_{1}$.

Definition 2.3. An equilibrium $K$ is globally asymptotically stable, if it is a global attractor for all solutions of (2.2)-(2.3) with the initial conditions in the open set $Q_{0} \subset \mathbb{R}^{n}$ and it is also locally uniformly stable.

Theorem 2.4. Suppose that $F(t, 0, \ldots, 0) \equiv 0, Q_{0} \subset \mathbb{R}^{n}$ is an open set, such that $0 \in Q_{0}$, an open set $Q \subset \mathbb{R}^{n}$ attracts all solutions of system (2.2)-(2.3) with the initial conditions in $Q_{0}$. Suppose also that there exists a nonnegative sequence $\lambda_{k}(1 \leq k \leq m)$ such that for any $u_{k} \in Q$ the following inequalities hold:

$\lim _{t \rightarrow \infty} \sup \left\|F\left(t, u_{1}, \ldots, u_{m}\right)\right\| \leq \sum_{k=1}^{m} \lambda_{k}\left\|u_{k}\right\|, \sum_{k=1}^{m} \lambda_{k}<\lim _{t \rightarrow \infty} \inf \left(-\mu_{A}(t)\right)$.

Then the trivial solution is the global attractor for all solutions of (2.2)-(2.3) with the initial conditions in $Q_{0}$.

If system is written in a scalar form, it is not always suitable to transform it to a vector form. In this case sometimes one can use a notion of Volterra operator.

We denote the space $C\left[t_{0}, \infty\right)$ of continuous bounded vector functions, and the space $L_{\infty}\left[t_{0}, \infty\right)$ of measurable essentially bounded vector functions, where both spaces are Banach spaces with usual supnorm.

Definition 2.5. Suppose $B_{1}$ and $B_{2}$ are two vector functional spaces on $\left[t_{0}, \infty\right)$. We say that operator $T: B_{1} \rightarrow B_{2}$ is a causal or Volterra operator if for any $t_{1}>t_{0}$ equality $x(t)=y(t), t \in\left[t_{0}, t_{1}\right]$ implies $(T x)(t)=(T y)(t), t \in\left[t_{0}, t_{1}\right]$.

We illustrate how a nonautonomous Nicholson-type model

$$
\begin{aligned}
& \dot{x_{1}}(t)=-a_{1}(t) x_{1}(t)+b_{1}(t) x_{2}(t)+c_{1}(t) x_{1}\left(h_{1}(t)\right) \exp \left(-x_{1}\left(h_{1}(t)\right)\right), \\
& \dot{x_{2}}(t)=-a_{2}(t) x_{2}(t)+b_{2}(t) x_{1}(t)+c_{2}(t) x_{2}\left(h_{2}(t)\right) \exp \left(-x_{2}\left(h_{2}(t)\right)\right)
\end{aligned}
$$

with the following initial value problem

$$
x(t)=\varphi(t)=\left\{\varphi_{1}(t), \varphi_{2}(t)\right\}^{T}, t<t_{0}, x\left(t_{0}\right)=x_{0},
$$

can be written in a compact form, using causal(Volterra) operators.

Suppose $a_{i}, b_{i}, c_{i}, i=1,2$ are essentially bounded on $[0, \infty)$ functions, $h_{k}, k=1, \ldots, m$ are measurable functions, $0 \leq t-h_{k}(t) \leq \tau, \varphi(t), t<t_{0}$ is a continuous vector-function.

$$
\begin{aligned}
& \text { Let } x=\left\{x_{1}, x_{2}\right\}^{T}, \\
& A(t)=\left[\begin{array}{cc}
-a_{1}(t) & b_{1}(t) \\
b_{2}(t) & -a_{2}(t)
\end{array}\right], \\
& x_{h}(t)=\left\{\begin{array}{ll}
x(h(t)) & t \geq t_{0} \\
0 & t<t_{0}
\end{array}, \varphi^{h}(t)= \begin{cases}\varphi(h(t)) & t \leq t_{0}, \\
0 & t>t_{0}\end{cases} \right. \\
& \left(F_{\varphi} x\right)(t)=\left\{c_{1}(t)\left[x_{1 h_{1}}(t)+\varphi_{1}^{h_{1}}(t)\right] \exp \left(-\left[x_{1 h_{1}}(t)+\varphi_{1}^{h_{1}}(t)\right]\right),\right. \\
& \left.c_{2}(t)\left[x_{2 h_{2}}(t)+\varphi_{2}^{h_{2}}(t)\right] \exp \left(-\left[x_{2 h_{2}}(t)+\varphi_{2}^{h_{2}}(t)\right]\right)\right\}^{T} .
\end{aligned}
$$

Then $F_{\varphi}: C\left[t_{0}, \infty\right) \rightarrow L_{\infty}\left[t_{0}, \infty\right)$ is a bounded nonlinear Volterra operator and system (2.4)-(2.5) has the operator form

$$
\frac{d x}{d t}=A(t) x(t)+\left(F_{\varphi} x\right)(t), t \geq t_{0}, x\left(t_{0}\right)=x_{0} .
$$


Let us fix $\tau>0$ and discuss the global stability conditions for the operator equation (2.9), where for any $t_{0} \geq 0$ and continuous vector function $\varphi:\left[t_{0}-\tau, t_{0}\right] \rightarrow \mathbb{R}^{n}$ operator $F_{\varphi}: C\left[t_{0}, \infty\right) \rightarrow L_{\infty}\left[t_{0}, \infty\right)$ is a bounded Volterra operator.

Theorem 2.6. Suppose that $\left(F_{\varphi} 0\right)(t) \equiv 0$ for $\varphi=0, Q_{0} \subset \mathbb{R}^{n}$ is an open set, such that $0 \in Q_{0}$, an open set $Q \subset \mathbb{R}^{n}$ attracts all solutions of (2.9) with the initial conditions in $Q_{0}$. Suppose also that there exist numbers $\lambda>0, \tau>0$ such that for any $x \in C\left[t_{0}, \infty\right), x(t) \in Q$ and $x\left(t_{0}\right) \in Q_{0}$ the following inequalities hold for sufficiently large $t$ :

$$
\left\|\left(F_{\varphi} x\right)(t)\right\| \leq \lambda \sup _{t-\tau \leq \xi \leq t}\|x(\xi)\|, \lambda<\alpha:=\lim _{t \rightarrow \infty} \inf \left(-\mu_{A}(t)\right),
$$

where $\lambda$ and $\tau$ do not depend on $\varphi$. Then the trivial solution is a global attractor for all solutions of (2.9) with initial conditions in $Q_{0}$.

As an application of the previous theorem consider Nicholson system with proportional coefficients

$$
\begin{aligned}
& \dot{x_{1}}(t)+r_{1}(t)\left(a_{1} x_{1}(t)-b_{1} x_{2}(t)-c_{1} x_{1}\left(h_{1}(t)\right) \exp \left(-x_{1}\left(h_{1}(t)\right)\right)\right)=0, \\
& \dot{x_{2}}(t)+r_{2}(t)\left(a_{2} x_{2}(t)-b_{2} x_{1}(t)-c_{2} x_{2}\left(h_{2}(t)\right) \exp \left(-x_{2}\left(h_{2}(t)\right)\right)\right)=0,
\end{aligned}
$$

$r_{i}(t)$ are measurable essentially bounded on $[0, \infty)$ nonnegative functions; and $a_{i}>0, b_{i}>0, c_{i}>0$, are constants.

Denote $Q_{i}=\lim \sup _{t \rightarrow \infty} r_{i}(t)$ and $q_{i}=\liminf _{t \rightarrow \infty} r_{i}(t)$.

Theorem 2.7. Suppose that a positive internal equilibrium $\left(x_{1}^{*}, x_{2}^{*}\right)$ of system (2.10) exists and for some $\epsilon>0$

$$
\begin{aligned}
& \max \left\{Q_{1} c_{1} e^{-2}, Q_{2} c_{2} e^{-2}, Q_{1} c_{1} e^{-x_{1}^{*}}-\epsilon, Q_{2} c_{2} e^{-x_{2}^{*}}-\epsilon, Q_{1} c_{1} e^{-x_{1}^{*}}\left|1-x_{1}^{*}\right|, Q_{2} c_{2} e^{-x_{2}^{*}}\left|1-x_{2}^{*}\right|\right\}< \\
& <\min \left\{q_{1}\left(a_{1}-b_{1}\right), q_{2}\left(a_{2}-b_{2}\right)\right\} .
\end{aligned}
$$

Then this equilibrium is globally asymptotically stable.

\subsection{M-matrix}

M-matrix method is widly used for all classes of systems of differential equations including FDE.

A matrix $B=\left(b_{i j}\right)_{i, j=1}^{m}$ is called a (non-singular) $M$-matrix if $b_{i j} \leq 0, i \neq j$ and one of the following equivalent conditions holds:

- there exists a positive inverse matrix $B^{-1}>0$;

- the principal minors of matrix $B$ are positive.

Consider first several known results.

In the paper [4] the authors consider the autonomous system for the system

$\dot{x}_{l}(t)=-\sum_{j=1}^{m} a_{i j} x_{j}\left(t-\tau_{i j}\right), i=1, \ldots, m$,

where $\tau_{i j} \geq 0$, the following result holds (below, $a_{+}$denotes the positive part of $a$, i.e., $a_{+}=\max \{a, 0\}$ ).

Theorem. Let

$0<a_{i i} \tau_{i i}<1+1 / e, i=1, \ldots, m$

and let the $m \times m$ matrix $H$ with components

$$
h_{i j}= \begin{cases}\left(\frac{1-\left(a_{i i} \tau_{i i}-1 / e\right)_{+}}{1+\left(a_{i i} \tau_{i i}-1 / e\right)_{+}}\right) a_{i i}, & i=j, \\ -\left|a_{i j}\right|, & i \neq j,\end{cases}
$$

$i, j=1, \ldots, m$ be a non-singular $M$-matrix. Then, system (2.11) is asymptotically stable for any selection of delays $\tau_{i j}, i \neq j, i, j=1, \ldots, m$.

In the paper [5] the authors consider the non-autonomous system

$$
\dot{x}_{l}(t)=-\sum_{j=1}^{m} a_{i j}(t) x_{j}\left(h_{i j}(t)\right), i=1, \ldots, m,
$$

where $t \in\left[t_{0}, \infty\right), t_{0} \in \mathbb{R}, a_{i j}(t), h_{i j}(t)$ are continuous functions, $h_{i j}(t) \leq t$, and $h_{i j}(t)$ are monotone increasing functions such that $\lim _{t \rightarrow \infty} h_{i j}(t)=\infty, i, j=1, \ldots, m$.

Theorem. Assume that, for $t \geq t_{0}$, there exist non-negative numbers $b_{i j}, i, j=1, \ldots, m, i \neq j$ such that $\left|a_{i j}(t)\right| \leq b_{i j} a_{i i}(t), i, j=1, \ldots, m, i \neq j, a_{i i}(t) \geq 0$ and

$$
\int^{\infty} a_{i i}(s) d s=\infty, d_{i}=\lim _{t \rightarrow \infty} \sup \int_{h_{i i}(t)}^{t} a_{i i}(s) d s<3 / 2, i=1, \ldots m .
$$

Let $\tilde{B}=\left(\tilde{b}_{i j}\right)_{i, j=1}^{m}$ be an $m \times m$ matrix with entries $\tilde{b}_{i i}=1, i=1, \ldots, m$ and, for $i \neq j, i, j=1, \ldots, m$, 


$$
\tilde{b}_{i j}=\left\{\begin{array}{l}
-\left(\frac{2+d_{i}^{2}}{2-d_{i}^{2}}\right) b_{i j}, \text { if } d_{i}<1, \\
-\left(\frac{1+2 d_{i}}{3-2 d_{i}}\right) b_{i j}, \text { if } d_{i} \geq 1 .
\end{array}\right.
$$

If $\tilde{B}$ is a nonsingular $M$-matrix, then system (2.12) is asymptotically stable.

We considered more general systems then previous ones and obtained new results which are independent on known ones.

The following results were obtained in the paper [6].

Consider for any $t_{0} \geq 0$ the system of delay differential equations

$$
\dot{x}_{i}(t)=-a_{i}(t) x_{i}\left(h_{i}(t)\right)+\sum_{j=1}^{m} F_{i j}\left(t, x_{j}\left(g_{i j}(t)\right)\right), t \geq t_{0}, i=1, \ldots, m,
$$

with the initial conditions

$$
x_{i}(t)=\varphi_{i}(t), t_{0}-\sigma \leq t<t_{0}, x_{i}\left(t_{0}\right)=x_{i}^{0},
$$

where $\sigma>0$ is denoted bellow in (a4) under the following assumptions:

(a1) $a_{i}$ are Lebesgue measurable essentially bounded on $[0, \infty)$ functions, $0<\alpha_{i} \leq a_{i}(t) \leq A_{i}$ almost everywhere (a.e.);

(a2) $F_{i j}(t, \cdot)$ are continuous functions, $F_{i j}(\cdot, u)$ are measurable locally essentially bounded functions, $\left|F_{i j}(t, u)\right| \leq L_{i j}|u|$, a.e. $t \geq 0$;

(a3) $h_{i}, g_{i j}$ are Borel measurable functions, $0 \leq t-h_{i}(t) \leq \tau_{i}, 0 \leq t-g_{i j}(t) \leq \sigma_{i j}$;

(a4) $\varphi_{i}$ are continuous functions on $\left[t_{0}-\sigma, t_{0}\right]$, where

$\sigma=\max \left\{\tau_{k}, \sigma_{i j} \mid k, i, j=1, \ldots, m\right\}$.

Assume that conditions $(a 1)-(a 4)$ hold for problem (2.13), (2.14) and its modifications, and the problem has a unique solution.

We will use some traditional notations. A matrix $B=\left(b_{i j}\right)_{i, j=1}^{m}$ is nonnegative if $b_{i j} \geq 0$ and positive if $b_{i j}>0, i, j=1, \ldots, m$; $\|a\|$ is an arbitrary fixed norm of a column vector $a=\left(a_{1}, \ldots, a_{m}\right)^{T}$ in $\mathbb{R}^{m}$; $\|B\|$ is the corresponding matrix norm of a matrix $B,|a|=\left(\left|a_{1}\right|, \ldots,\left|a_{m}\right|\right)^{T}$ and $|B|=\left(\mid b_{i j \mid}\right)_{i, j=1}^{m}$.

Problem (2.13), (2.14) has a unique global solution on $\left[t_{0} ; \infty\right)$, if, for example, we assume along with $(a 1)-(a 4)$ that functions $F_{i j}(t ; u)$ are locally Lipschitz in $u$. The following classical definition of an $M$-matrix will be used.

Definition 2.8. A matrix $B=\left(b_{i j}\right)_{i, j=1}^{m}$ is called a (non-singular) $M$-matrix if $b_{i j} \leq 0 ; i \neq j$ and one of the following equivalent conditions holds:

- there exists a positive inverse matrix $B^{-1}>0$;

- the principal minors of matrix $B$ are positive.

Lemma 2.9. $B$ is an M-matrix if $b_{i j} \leq 0 ; i \neq j$ and at least one of the following conditions holds:

1) $b_{i i}>\sum_{j \neq i}\left|b_{i j}\right| ; i=1, \ldots, m$;

2) $b_{j j}>\sum_{i \neq j}\left|b_{i j}\right| ; j=1, \ldots, m$;

3) there exist positive numbers $\xi_{i} ; i=1, \ldots, m$ such that $\xi_{i} b_{i i}>\sum_{j \neq i} \xi_{j}\left|b_{i j}\right|, i=1, \ldots, m$;

4) there exist positive numbers $\xi_{i} ; i=1, \ldots, m$ such that $\xi_{j} b_{j j}>\sum_{i \neq j} \xi_{i}\left|b_{i j}\right|, j=1, \ldots, m$.

Definition 2.10. System (2.13) is globally exponentially stable if there exist constants $M>0$ and $\lambda>0$ such that for any solution $X(t)$ of problem (2.13), (2.14) the inequality

$$
\|X(t)\| \leq M e^{-\lambda\left(t-t_{0}\right)}\left(\left\|x\left(t_{0}\right)\right\|+\sup _{t<t_{0}}\|\varphi(t)\|\right)
$$

holds, where $M$ and $\lambda$ do not depend on $t_{0}$.

We define matrix $C$ as follows

$$
C=\left(c_{i j}\right)_{i, j=1}^{m}, \quad c_{i i}=1-\frac{A_{i}\left(A_{i}+L_{i i}\right) \tau_{i}+L_{i i}}{\alpha_{i}}, c_{i j}=-\frac{A_{i} L_{i j} \tau_{i}+L_{i j}}{\alpha_{i}}, i \neq j \text {. }
$$

Theorem 2.11. Suppose $C$ defined by (2.15) is an M-matrix. Then system (2.13) is globally exponentially stable.

As an application of this result we consider non-autonomous BAM (bidirectional associative memory) neural network model 


$$
\begin{aligned}
& \dot{x}_{l}(t)=r_{i}(t)\left(-a_{i} x_{i}\left(h_{i}^{(1)}(t)\right)+\sum_{j=1}^{n} a_{i j} f_{j}\left(y_{j}\left(l_{j}^{(2)}(t)\right)\right)+I_{i}\right), \\
& \dot{y}_{l}(t)=r_{i}(t)\left(-b_{i} y_{i}\left(h_{i}^{(2)}(t)\right)+\sum_{j=1}^{n} b_{i j} g_{j}\left(x_{j}\left(l_{j}^{(1)}(t)\right)\right)+J_{i}\right),
\end{aligned}
$$

$i=1, \ldots, n, t \geq 0$, with the initial conditions

$$
x_{i}(t)=\varphi_{i}(t), y_{i}(t)=\varphi_{i+n}(t), t<0, i=1, \ldots, n .
$$

We will say that a norm in $\mathbb{R}^{n}$ is monotone if $0 \leq a \leq b$ (a componentwise inequality, i.e. $0 \leq a_{i} \leq b_{i}$ for all $i$ ) implies $\|a\| \leq\|b\|$.

Consider the following algebraic system

$$
u_{i}=\sum_{j=1}^{m} F_{i j}\left(u_{j}\right), i=1, \ldots, m,
$$

where $\left|F_{i j}(u)-F_{i j}(v)\right| \leq L_{i j}|u-v|$.

Lemma 2.12. Let $r(L)$ be a spectral radius of the matrix $L=\left(L_{i j}\right)_{i, j=1}^{m}$. If $r(L)<1$ then system (2.18) has a unique solution.

Theorem 2.13. Suppose at least one of the following conditions holds:

1. $\max |\lambda(A)|<1$, where maximum is taken on all eigenvalues of matrix $A$.

2. $\max _{i} \sum_{j=1}^{n} \frac{\left|a_{i j}\right| L_{j}^{f}}{a_{i}}<1, \max _{i} \sum_{j=1}^{n} \frac{\left|b_{i j}\right| L_{j}^{g}}{b_{i}}<1$.

3. $\max _{j} \sum_{i=1}^{n} \frac{\left|a_{i j}\right| L_{j}^{f}}{a_{i}}<1, \max _{j} \sum_{i=1}^{n} \frac{\left|b_{i j}\right| L_{j}^{g}}{b_{i}}<1$.

4. $\sum_{i=1}^{n} \sum_{j=1}^{n}\left[\left(\frac{\left|a_{i j}\right| L_{j}^{f}}{a_{i}}\right)^{2}+\left(\frac{\left|b_{i j}\right| L_{j}^{g}}{b_{i}}\right)^{2}\right]<1$.

5. $\max |\lambda(\mathrm{B})|<1$, where maximum is taken on all eigenvalues of matrix $B$.

6. $\max _{i} \sum_{j=1}^{n} \frac{\left|a_{i j}\right| L_{j}^{f}}{b_{j}}<1, \max _{i} \sum_{j=1}^{n} \frac{\left|b_{i j}\right| L_{j}^{g}}{a_{j}}<1$.

7. $\max _{j} \sum_{i=1}^{n} \frac{\left|a_{i j}\right| L_{j}^{f}}{b_{j}}<1, \max _{j} \sum_{i=1}^{n} \frac{\left|b_{i j}\right| L_{j}^{g}}{a_{j}}<1$.

8. $\sum_{i=1}^{n} \sum_{j=1}^{n}\left[\left(\frac{\left|a_{i j}\right| L_{j}^{f}}{b_{j}}\right)^{2}+\left(\frac{\left|b_{i j}\right| L_{j}^{g}}{a_{j}}\right)^{2}\right]<1$.

Then system

$$
\begin{aligned}
& a_{i} x_{i}=\sum_{j=1}^{n} a_{i j} f_{j}\left(y_{j}\right)+I_{i}, \\
& b_{i} y_{i}=\sum_{j=1}^{n} b_{i j} g_{j}\left(x_{j}\right)+J_{i}
\end{aligned}
$$

has a unique solution and thus system (2.16) has a unique equilibrium.

Below, assume that system (2.16) has a unique equilibrium $\left(x^{*} ; y^{*}\right)$. To obtain a global stability condition for this equilibrium, consider the matrix $C_{B A M}=\left(c_{i j}\right)_{i, j=1}^{2 n}$, where

$$
\begin{aligned}
& c_{i i}=\left\{\begin{array}{c}
1-\frac{a_{i} R_{i}^{2} \tau_{i}^{(1)}}{\alpha_{i}}, i=1, \ldots, n, \\
1-\frac{b_{i-n} P_{i-n}^{2} \tau_{i-n}^{(1)}}{\beta_{i-n}}, i=n+1, \ldots, 2 n,
\end{array}\right. \\
& c_{i j}=\left\{\begin{array}{c}
-\frac{\left|a_{i, j-n}\right| R_{i} L_{j-n}^{f}\left(a_{i} R_{i} \tau_{i}^{(1)}+1\right)}{\alpha_{i} a_{i}}, i=1, \ldots, n, j=n+1, \ldots, 2 n, \\
-\frac{\left|b_{i-n, j}\right| P_{i-n} L_{j}^{g}\left(b_{i-n} P_{i-n} \tau_{i-n}^{(2)}+1\right)}{\beta_{i-n} b_{i-n}}, i=n+1, \ldots, 2 n, j=1, \ldots, n, \\
0, \text { otherwise. }
\end{array}\right.
\end{aligned}
$$

Theorem 2.14. Suppose matrix $C_{B A M}$ is an M-matrix. Then the equilibrium $\left(x^{*} ; y^{*}\right)$ of system (2.16) is globally exponentially stable.

Corollary 1. Suppose at least one of the following conditions holds: 
1. $\sum_{j=1}^{n} \frac{\left|a_{i j}\right| R_{i} L_{j}^{f}\left(a_{i} R_{i} \tau_{i}^{(1)}+1\right)}{\alpha_{i} a_{i}}<1-\frac{a_{i} R_{i}^{2} \tau_{i}^{(1)}}{\alpha_{i}}$,

$\sum_{j=1}^{n} \frac{\left|b_{i j}\right| P_{i} L_{j}^{g}\left(b_{i} P_{i} \tau_{i}^{(2)}+1\right)}{\beta_{i} b_{i}}<1-\frac{b_{i} P_{i}^{2} \tau_{i}^{(2)}}{\beta_{i}}, i=1, \ldots, n$.

2. $\sum_{i=1}^{n} \frac{\left|a_{i j}\right| R_{i} L_{j}^{f}\left(a_{i} R_{i} \tau_{i}^{(1)}+1\right)}{\alpha_{i} a_{i}}<1-\frac{b_{j} P_{j}^{2} \tau_{j}^{(2)}}{\beta_{j}}$,

$\sum_{i=1}^{n} \frac{\left|b_{i j}\right| P_{i} L_{j}^{g}\left(b_{i} P_{i} \tau_{i}^{(2)}+1\right)}{\beta_{i} b_{i}}<1-\frac{a_{j} R_{j}^{2} \tau_{j}^{(1)}}{\alpha_{j}}, j=1, \ldots, n$.

3. There exist positive numbers $\mu_{k} ; k=1, \ldots, 2 n$ such that

$\sum_{j=1}^{n} \frac{\mu_{j+n}\left|a_{i j}\right| R_{i} L_{j}^{f}\left(a_{i} R_{i} \tau_{i}^{(1)}+1\right)}{\alpha_{i} a_{i}}<\mu_{i}\left(1-\frac{a_{i} R_{i}^{2} \tau_{i}^{(1)}}{\alpha_{i}}\right)$,

$\sum_{j=1}^{n} \frac{\mu_{j}\left|b_{i j}\right| P_{i} L_{j}^{g}\left(b_{i} P_{i} \tau_{i}^{(2)}+1\right)}{\beta_{i} b_{i}}<\mu_{i+n}\left(1-\frac{b_{i} P_{i}^{2} \tau_{i}^{(2)}}{\beta_{i}}\right)$,

$(i=1, \ldots, n)$.

4. There exist positive numbers $\mu_{k} ; k=1, \ldots, 2 n$ such that

$\sum_{i=1}^{n} \frac{\mu_{i+n}\left|a_{i j}\right| R_{i} L_{j}^{f}\left(a_{i} R_{i} \tau_{i}^{(1)}+1\right)}{\alpha_{i} a_{i}}<\mu_{j}\left(1-\frac{b_{j} P_{j}^{2} \tau_{j}^{(2)}}{\beta_{j}}\right)$,

$\sum_{i=1}^{n} \frac{\mu_{j}\left|b_{i j}\right| P_{i} L_{j}^{g}\left(b_{i} P_{i} \tau_{i}^{(2)}+1\right)}{\beta_{i} b_{i}}<\mu_{j+n}\left(1-\frac{a_{j} R_{j}^{2} \tau_{j}^{(1)}}{\alpha_{j}}\right)$,

$(j=1, \ldots, n)$.

Then the equilibrium $\left(x^{*} ; y^{*}\right)$ of system (2.16) is globally exponentially stable.

\section{SCALAR FORM SYSTEM WITHOUT APPLICATIONS OF SPECIAL MATRIX}

The results of this part were obtained in the paper [7].

The aim of this part is to obtain easily checked explicit exponential stability conditions for the following non-autonomous linear delay differential system

$$
\dot{x}_{i}(t)=-\sum_{j=1}^{m} \sum_{k=1}^{r_{i j}} a_{i j}^{k}(t) x_{j}\left(h_{i j}^{k}(t)\right), i=1, \ldots, m,
$$

where $t \geq 0, m$ and $r_{i j}, i, j=1, \ldots, m$ are natural numbers, coefficients $a_{i j}^{k}:[0, \infty) \infty \rightarrow \mathbb{R}$ and delays $h_{i j}^{k}:[0, \infty) \infty \rightarrow \mathbb{R}$ are measurable functions.

Define auxiliary functions

$a_{i}(t):=\sum_{k=1}^{r_{i i}} a_{i i}^{k}(t), i=1, \ldots, m, t \in[0, \infty)$.

Theorem 3.1. Assume that, for $t \geq t_{0}$, and$$
a_{i}(t) \geq a_{0}>0, i=1, \ldots, m
$$

$\max _{i=1, \ldots, m} \operatorname{ess} \sup _{t \geq t_{0}} \frac{1}{a_{i}(t)} \times$

$\times\left[\sum_{k=1}^{r_{i i}}\left|a_{i i}^{k}(t)\right| \int_{\max \left\{0, h_{i i}^{k}(t)\right\}}^{t} \sum_{j=1}^{m} \sum_{l=1}^{r_{i j}}\left|a_{i j}^{l}(s)\right| d s+\sum_{\substack{j=1 \\ j \neq i}}^{m} \sum_{k=1}^{r_{i j}}\left|a_{i j}^{k}(t)\right|\right]<1$.

Then, system (3.3) is uniformly exponentially stable.

In our new paper which is now on preparation we improve the result of the previous paper by replacing the constant 1 by the constant $1+\frac{1}{e}$ which is one of the best known constants in stability investigations.

\section{References}

1. Gil M. Stability of Finite and Infinite Dimensional Systems, Kluwer Academic Publishers, 1998. DOI: 10.1007/978-1-4615-5575-9

2. Idels L., Kipnis M. Stability Criteria for a Nonlinear Nonautonomous System with Delays. Appl. Math. Model., 2009, vol. 33, no. 5, pp. 2293-2297. 
3. Berezansky L., Idels L., Troib L. Global Dynamics of One Class of Nonlinear Nonautonomous Systems with Time-Varying Delays. Nonlinear Anal., 2011, vol. 74, no. 18, pp. 7499-7512.

4. Györi I., Hartung F., Turi J., Preservation of Stability in Delay Equations under Delay Perturbations, J. Math. Anal. Appl., 1998, vol. 220, pp. 290-312. DOI: 10.1006/jmaa.1997.5883

5. So Joseph W.-H., Tang X.H., Zou Xingfu. Global Attractivity for Non-Autonomous Linear Delay Systems, Funkcial. Ekvac., 2004, vol. 47, no. 1, pp. 25-40.

6. Berezansky L., Idels L., Troib L. Global Dynamics of Nicholson-Type Delay Systems with Applications. Nonlinear Anal. Real World Appl., 2011, vol. 12, no. 1, pp. 436-445.

7. Berezansky L., Diblik J., Svoboda Z., Smarda Z. Simple Uniform Exponential Stability Conditions for a System of Linear Delay Differential Equations. Appl. Math. Comput., 2015, vol. 250, pp. 605-614. DOI: 10.1016/j.amc.2014.10.117

Received 25 February 2017

удк 51-77

DOI: $10.14529 /$ ctcr170214

\title{
ГЛОБАЛЬНАЯ ЭКСПОНЕНЦИАЛЬНАЯ УСТОЙЧИВОСТЬ ДЛЯ ДИФФЕРЕНЦИАЛЬНЫХ СИСТЕМ С НЕЛИНЕЙНЫМИ ЗАДЕРЖКАМИ
}

\author{
Л. Березанский \\ Университет имени Бен-Гуриона, Беэр-Шева, Израиль
}

\begin{abstract}
Даётся обзор последних результатов по глобальной стабильности для нелинейного уравнения функционального дифференциала. Такие уравнения включают дифференциальные задержки, интегро-дифференциальные уравнения и уравнения с распределенным запаздыванием и применяются в качестве математических моделей в области динамики народонаселения и других наук. Также рассмотрены методы, используемые для изучения глобальной стабильности: построение функционалов Ляпунова, применение специальных матриц, таких как М-матрица или специальных матричных функций, таких как матричная мера, метод матричных неравенств, которые очень популярны в работах по теории контроля, метод неподвижной точки и использование понятия нелинейного оператора Вольтерра.

Ключевые слова: глобальная стабильность, функционал Ляпунова, матричная мера, метод матричных неравенств, нелинейный оператор Вольтерра.
\end{abstract}

Березанский Леонид, отделение математики, Университет имени Бен-Гуриона, Беэр-Шева, Израиль; brznsky@cs.bgu.ac.il.

Поступила в редакцию 25 февраля 2017 2.

\section{ОБРАЗЕЦ ЦИТИРОВАНИЯ}

Berezansky, L. Global Exponential Stability for Nonlinear Delay Differential Systems / L. Berezansky // Вестник ЮУрГУ. Серия «Компьютерные технологии, управление, радиоэлектроника». - 2017. - Т. 17, № 2. C. $149-155$. DOI: $10.14529 /$ ctcr170214

\section{FOR CITATION}

Berezansky L. Global Exponential Stability for Nonlinear Delay Differential Systems. Bulletin of the South Ural State University. Ser. Computer Technologies, Automatic Control, Radio Electronics, 2017, vol. 17 , no. 2, pp. 149-155. DOI: 10.14529/ctcr170214 